\title{
Le statut officiel et social du komi sur le territoire de la République des Komis : histoire et situation actuelle
}

The official and social status of Komi language on the territory of Komi Republic (history and present situation)

ГОСУДАРСТВЕННЫЙ И ОБЩЕСТВЕННЫЙ СТАТУС КОМИ ЯЗЫКА НА ТЕРРИТОРИИ

РЕСПУБЛИКИ КОМИ (ИСТОРИЯ И СОВРЕМЕННОСТЬ)

КОМИ РЕСПУБЛИКА МУТАСЫН КОМИ КЫВЛӦН КАНМУ ДА ВОЙТЫРКОСТСА

ТӧДЧАНЛУН (ИСТОРИЯ ДА ӧНіЯ КАД)

\section{Ol'ga Kuzivanova et Marina Fedina}

Traducteur : Antoine Chalvin

\section{OpenEdition Journals}

Édition électronique

URL : https://journals.openedition.org/efo/1956

DOI : $10.4000 /$ efo. 1956

ISSN : 2275-1947

Éditeur

INALCO

\section{Édition imprimée}

Date de publication : 1 décembre 2013

ISBN : 978-2-343-04446-0

ISSN : 0071-2051

Référence électronique

Ol'ga Kuzivanova et Marina Fedina, «Le statut officiel et social du komi sur le territoire de la

République des Komis : histoire et situation actuelle », Études finno-ougriennes [En ligne], 45 | 2013, mis en ligne le 27 janvier 2015, consulté le 08 juillet 2021. URL : http://journals.openedition.org/efo/1956 ; DOI : https://doi.org/10.4000/efo.1956

Ce document a été généré automatiquement le 8 juillet 2021.

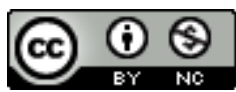

Études finno-ougriennes est mis à disposition selon les termes de la Licence Creative Commons Attribution - Pas d'Utilisation Commerciale 4.0 International. 


\section{Le statut officiel et social du komi sur le territoire de la République des Komis : histoire et situation actuelle}

The official and social status of Komi language on the territory of Komi Republic (history and present situation)

ГОСУДАРСТВЕННЫЙ И ОБЩЕСТВЕННЫЙ СТАТУС КОМИ ЯЗЫКА НА ТЕРРИТОРИИ

РЕСПУБЛИКИ КОМИ (ИСТОРИЯ И СОВРЕМЕННОСТЬ)

КОМИ РЕСПУБЛИКА МУТАСЫН КОМИ КЫВЛӦН КАНМУ ДА ВОЙТЫРКОСТСА

ТӧДЧАНЛУН (ИСТОРИЯ ДА ӦНіЯ КАД)

Ol'ga Kuzivanova et Marina Fedina

Traduction : Antoine Chalvin

1 Dans la Fédération de Russie, pays pluriethnique de 174 groupes nationaux, la mise en œuvre d'une politique des nationalités réfléchie a toujours été un sujet à l'ordre du jour. L'un des instruments d'harmonisation des relations interethniques est la politique ethnolinguistique.

2 Les Komis sont un des peuples finno-ougriens vivant au nord-est de la partie européenne de la Russie. En 1921 fut créé au sein de la Fédération de Russie un oblast autonome komi, incluant les territoires peuplés majoritairement de Komis (Zyriènes). À cette époque, les Komis représentaient plus de $90 \%$ de la population de la région. Cela posa immédiatement la question du statut de la langue komie sur ce territoire.

\section{Aperçu historique}

3 L'élite komie, composée à cette époque de communistes (qui avaient pris part à la guerre civile de 1918-1921), des employés des organes soviétiques et des membres de l'intelligentsia (surtout enseignants), accueillit avec enthousiasme les mesures de la 
politique ethnolinguistique des bolcheviks, visant à instaurer l'usage des langues autochtones dans l'enseignement scolaire, l'administration et la vie publique.

4 En novembre 1923, le comité de l'oblast du $\operatorname{PCR}(b)^{1}$ créa auprès du comité exécutif de l'oblast ${ }^{2}$ une commission spéciale chargée d'élaborer et de mettre en œuvre la "zyriénisation $~^{3}$, qui organisa l'introduction du komi dans les activités administratives régionales, le travail des établissements d'enseignement en langue autochtone, et encouragea la publication d'œuvres littéraires en komi. Son activité couvrait donc presque tous les domaines culturels et linguistiques. Elle comprenait un centre régional et des commissions de district subordonnées, et un réseau de représentants accrédités au sein des institutions.

5 Un décret du Comité exécutif central panrusse du 14 avril 1924 « sur l'introduction des langues locales dans les activités administratives des organes étatiques des Républiques et oblasts nationaux" confirma la ligne du gouvernement de la RSFSR, visant «l'adaptation de l'appareil soviétique au mode de vie des populations autochtones des Républiques et oblasts nationaux et l'engagement actif de ces populations dans la construction soviétique » (Popov 2004, p. 302). La commission de zyriénisation prépara la définition du statut de la langue komie. En octobre 1924, le $4^{\mathrm{e}}$ Congrès régional des soviets - organe représentatif suprême du pouvoir politique de l'oblast autonome komi - considéra «comme langues officielles, sur le territoire de l'oblast autonome komi, le komi et le russe » (Popov 2004, p. 302).

6 Sur cette base fut élaborée une "Instruction relative à l'introduction de la langue komie dans les administrations et établissements publics, les entreprises et les organisations de l'oblast autonome komi ». Elle prescrivait l'usage du komi dans les activités administratives, à l'exception de la correspondance extérieure (avec des établissements situés hors de l'oblast) et des localités peuplées de Russes. Le contrôle de l'application de cette décision fut confié à la Commission régionale des ouvriers et paysans (CROP), qui disposait des plus larges pouvoirs. La vérification de l'application des directives était réalisée de façon systématique, et les conclusions étaient publiées dans les journaux. Citons un extrait du rapport du collège de la CROP de 1929: «Le collège a jugé devoir demander au Comité exécutif de l'oblast... d'ordonner à titre complémentaire que la correspondance entre les institutions, les organisations, les établissements, tant dans le centre régional que dans les centres de district et les districts ruraux, se fasse obligatoirement en komi ; de publier sans délai les noms des dirigeants signant des correspondances en russe et de leur adresser des rappels à l'ordre ; s'il est établi que la correspondance est systématiquement rédigée en russe et qu'un dirigeant doit être plusieurs fois rappelé à l'ordre, de considérer une telle infraction comme délibérée et de la sanctionner par des mesures pouvant aller jusqu'à la révocation " (Osipov 1929, p. 8).

7 Dans les procédures judiciaires, il était réellement possible d'utiliser la langue komie. Dans les années vingt et trente, la directive relative aux tribunaux était assez stricte : toutes les affaires judiciaires dont les parties étaient des Komis devaient être conduites en komi d'un bout à l'autre. Les miliciens des villages, des villes et des districts, mais aussi les juges d'instruction, devaient mener leurs enquêtes en komi dans tous les cas où l'affaire, en totalité ou en majeure partie, concernait la population komie (Osipov 1929, p. 9). Cette directive fut dans l'ensemble bien appliquée, même si des complications se firent jour, par exemple dans les cas où l'enquêteur ou le juge ne connaissaient pas le komi. 
8 Les actes législatifs ultérieurs de nature constitutionnelle dégradèrent le statut de la langue komie sur le territoire autonome. Ainsi, dans la Constitution de la République socialiste soviétique autonome komie ${ }^{4}$ de 1937, cinq articles mentionnent la langue komie :

Article 24. Les lois adoptées par le Soviet suprême de la RSSA komie sont publiées en komi et en russe après signature par le président et par le secrétaire du Praesidium du Soviet suprême de la RSSA komie (Tvoi 2004, p. 11) ;

Article 82. Les procédures judiciaires dans la RSSA komie se déroulent en komi, en garantissant aux personnes qui ne maîtrisent pas cette langue la possibilité de prendre connaissance pleine et entière du dossier par l'intermédiaire d'un traducteur, ainsi que le droit de s'exprimer devant le tribunal dans leur langue maternelle (Tvoi 2004, p. 23);

Article 92. Les citoyens de la RSSA komie ont droit à l'éducation. Ce droit est garanti par l'enseignement élémentaire général et obligatoire, par la gratuité de l'enseignement, y compris l'enseignement supérieur, par un système de bourses d'État accordées à la majorité des étudiants des établissements d'enseignement supérieur, par l'enseignement scolaire en langue maternelle, par l'organisation, dans les usines, les sovkhozes, les stations de machines et de tracteurs et les kolkhozes, de la formation gratuite des travailleurs dans les domaines de la production, de la technique et de l'agronomie (Tvoi 2004, p. 25) ;

Article 115. Le blason officiel de la RSSA komie est celui de la RSFSR, qui représente une faucille et un marteau en or disposés en croix, le manche vers le bas, sur un fond rouge, dans les rayons du soleil et encadrés par des épis, avec les inscriptions «RSFSR » et « Prolétaires de tous les pays, unissez-vous ! dans les langues russe et komie, avec, en complément, sous l'inscription «RSFSR », en lettres plus petites, l'inscription « RSSA komie » en russe et en komi ;

Article 116. Le drapeau officiel de la RSSA komie est celui de la RSFSR, constitué d'une étoffe rouge dans le coin supérieur gauche de laquelle, du côté de la hampe, est écrit en lettres dorées "RSFSR », et, au-dessous, en caractères plus petits, la mention «RSSA komie » en russe et en komi (Tvoi 2004, p. 31).

9 La Constitution de 1937 ne contient aucune clause posant le statut officiel de la langue komie à égalité avec le russe sur le territoire de la RSSA komie. Cette clause avait été écartée pendant la phase d'élaboration du projet de constitution de 1936 (Popov \& Napalkov 2004, p. 38).

La Constitution suivante de la RSSA komie, adoptée en 1978, mentionnait le statut de la langue komie dans trois articles :

L'article 39 (qui correspondait à l'article 92 de la Constitution de 1937), dans lequel la formule « enseignement scolaire en langue maternelle » était remplacée par « la possibilité d'un enseignement scolaire en langue maternelle » (Cypanov, p. 89) ; L'article 148 (article 82 de la Constitution de 1937) était rédigé comme suit : Les procédures judiciaires dans la RSSA komie se déroulent en komi, en russe ou dans la langue de la majorité de la population de la localité concernée. Les parties ne maîtrisant pas la langue de la procédure ont le droit de prendre connaissance pleine et entière des documents liés à l'affaire et de s'exprimer devant le tribunal dans leur langue maternelle, par l'intermédiaire d'un traducteur (Tvoi, p. 120) ;

L'article 157 stipule que la devise « Prolétaires de tous les pays, unissez-vous ! » doit être écrite en russe et en komi.

11 Dans la loi de la RSSA komie du 21 mars 1980, l'article 39 a été supprimé. Seuls ont été conservés les articles 148 et 157. 


\section{Le statut actuel de la langue komie}

12 La politique ethnolinguistique mise en œuvre sur le territoire de la RSSA komie a été déterminée par de nombreux facteurs, notamment la composition nationale de la République. En quelques décennies d'autonomie komie au sein de la Fédération de Russie, la proportion des principales nationalités présentes sur le territoire s'est modifiée. En 1939, les Komis ne représentaient plus que 72,5\% de la population de la RSSA komie. Les changements ultérieurs de la composition nationale de la République sont présentés dans le tableau 1 ci-dessous.

Tableau 1. Proportion des Komis et des Russes dans la population de la République des Komis (d'après les recensements de la population, en pourcentage)

\begin{tabular}{|l|l|l|l|l|l|}
\hline & 1959 & 1970 & 1979 & 1989 & 2002 \\
\hline Komis & 30,1 & 28,6 & 25,3 & 23,3 & 25,2 \\
\hline Russes & 48,6 & 53,1 & 56,7 & 57,7 & 59,6 \\
\hline
\end{tabular}

(Nacional'nyj 2005, p. 39).

$\mathrm{Du}$ fait que les Komis se sont retrouvés minoritaires sur leur territoire traditionnel, la question de la préservation de leur langue et de leur culture s'est posée de façon particulièrement aiguë. À la fin des années 1980 , le mouvement national komi a soulevé la question de la nécessité d'un soutien officiel à la langue et à la culture komies. Compte tenu de la nouvelle situation sociopolitique en Russie et dans la République komie, une politique officielle en matière de nationalités a commencé à être mise en place au sein de la République, et une attention particulière a été accordée aux questions relatives à la culture komie (Popov \& Nesterova 2006, p. 51-52).

En mai 1992 fut adoptée une loi sur les Langues officielles de la république des Komis, qui prévoyait l'utilisation du komi et du russe à divers degrés sur le territoire de la République. La langue komie retrouvait ainsi un statut officiel.

En février 1994 fut adoptée la Constitution de la République des Komis. Le statut officiel du komi fut confirmé par l'article 67 :

Les langues officielles de la République des Komis sont le komi et le russe. La République des Komis garantit aux peuples résidant sur son territoire le droit de conserver leur langue maternelle et la mise en place des conditions nécessaires à l'apprentissage et au développement de celle-ci (Tvoi 2004, p. 227).

L'article 3 de la Constitution est particulièrement important pour les Komis :

Le peuple komi est la source du statut étatique de la République des Komis. La politique de l'État vise à soutenir et à développer la langue, la culture et le mode de vie du peuple komi, conformément aux normes et aux principes internationaux relatifs aux peuples autochtones (Tvoi 2004, p. 204).

17 Une confirmation du statut de la langue komie figure également à l'article 83, selon lequel le chef de l'État de la République des Komis doit prêter serment dans les deux langues officielles (Tvoi 2004, p. 236).

18 Avec quelques corrections introduites dans la version de 2002, la loi sur les Langues officielles de la République des Komis est toujours en vigueur aujourd'hui. Le contrôle de son application a été dévolu au ministère de la Politique nationale de la République 
des Komis (de 1994 à 2003, ministère des Questions nationales; à partir de 2007, ministère de la Politique nationale; entre 2003 et 2007, les questions nationales relevaient du ministère de la Culture et de la politique nationale). Des rapports sur l'application de la loi sont systématiquement présentés devant les collèges du ministère. Une différence importante du contrôle linguistique pour la période actuelle réside dans l'absence de sanctions sévères en cas de non-respect des dispositions de la loi. Le caractère plus doux, mesuré et civilisé de la mise en œuvre de la politique linguistique constitue un avantage et une condition nécessaire au maintien d'une relative stabilité des relations interethniques au sein de la République des Komis, qui compte aujourd'hui plus de cent nationalités.

En avril 1994 a été créée une commission de terminologie et d'orthographe (CTO) rattachée au ministère des Questions nationales. Si la commission de zyriénisation possédait un centre régional et des sous-commissions de district, mais aussi tout un réseau de représentants au sein des institutions, l'actuelle CTO est une structure non gouvernementale composée de 22 personnes. Mais l'importance de ces deux commissions est indéniable. Elles ont toutes deux contribué de façon considérable au développement de la langue komie et à l'élargissement réel de ses fonctions au niveau officiel. La CTO a été très active pendant huit ans, après quoi son activité s'est progressivement réduite et s'est même pratiquement interrompue entre 2004 et 2008. Elle a retrouvé un nouveau souffle au début de l'année 2009, après le renouvellement de sa composition et la révision de ses objectifs et de ses missions.

Aujourd'hui, tous les actes normatifs adoptés par les autorités de la République des Komis sont publiés dans les deux langues. Il existe une revue spéciale intitulée «Bulletin des actes juridiques des organes étatiques de la République des Komis » (en komi: КОМИ РЕСПУБЛИКАСА ГОСУДАРСТВЕННӦЙ ВЛАСЬТ ОРГАНЪЯСЛӦН ИНДӧДтшӧктӧмъяс), qui alimente le portail Internet officiel de la République des Komis en langue komie.

21 La Constitution de la République des Komis a été traduite en komi, ainsi que les emblèmes officiels de la République. Les documents d'état civil sont rédigés dans les deux langues officielles, de même que les textes accompagnant les décorations décernées par les organes du pouvoir législatif et exécutif de la République, les diplômes des lauréats des prix d'État, les bulletins de vote pour les élections à différents niveaux. Aujourd'hui, tous les habitants de la Fédération de Russie peuvent adresser des demandes en komi aux autorités de la République et recevoir des réponses. Le komi est représenté de façon visible dans les enseignes des établissements et organisations, indépendamment de leur régime de propriété, ainsi que sur les plaques des rues et des places.

Conformément à l'article 9 de la loi sur les Langues officielles, les procédures judiciaires au sein de la République doivent être conduites en komi et en russe. Pourtant, dans la grande majorité des procédures, la préférence est donnée à la langue russe, sauf dans les cas où le procès est traduit en komi à la demande de personnes âgées.

Naturellement, du point de vue des résultats, la politique linguistique actuelle est très différente de celle des années vingt et trente du $\mathrm{xx}^{\mathrm{e}}$ siècle. E. A. Cypanov porte ainsi un jugement assez pessimiste sur l'efficacité de la politique actuelle: «L'utilisation du komi dans les administrations de l'État progresse avec une lenteur extrême, et les succès enregistrés dans ce domaine sont relativement modestes. On s'en rend compte tout particulièrement si l'on compare cette politique avec les mesures de 
«zyriénisation-indigénisation» des années vingt et trente, qui avaient été mises en œuvre très rapidement et de façon très efficace. Mais depuis lors, la composition nationale de la population s'est modifiée, la conscience nationale s'est affaiblie, des attitudes de rejet, d'indifférence ou de passivité à l'égard de sa propre nationalité sont apparues, et dans le choix de la langue de communication ou d'éducation des enfants prédomine aujourd'hui un pur et froid pragmatisme. L'utilisation du komi dans la sphère officielle se heurte à des difficultés financières et économiques. La difficulté principale est toutefois de nature psychologique, voire individuelle: les agents de l'administration ne veulent pas avoir affaire à la langue komie, et la majorité des fonctionnaires komis ne voient pas l'intérêt d'utiliser une seconde langue dans le cadre de leur travail (Cypanov 2006, p. 152).

Les causes de cette utilisation insuffisante et non systématique du komi dans la sphère officielle et les affaires sont complexes, mais, selon nous, les principales raisons sont une demande sociale insuffisante et l'absence d'incitation à utiliser la langue. Des mesures coercitives, telles que celles qui avaient été adoptées à l'époque de la zyriénisation, ne donneraient aujourd'hui aucun résultat, comme en témoigne éloquemment l'objection du Parquet de la République des Komis à l'égard de la proposition de quelques députés de rendre la connaissance du komi obligatoire pour les fonctionnaires. En la matière, on pourrait mentionner un exemple positif : le ministère de la Culture de la République des Komis a publié en 2008 des instructions selon lesquelles les agents des établissements publics culturels et artistiques qui connaissent le komi et l'utilisent dans le cadre de leur travail reçoivent une prime pouvant aller jusqu'à $25 \%$ de leur salaire. Cette mesure a déjà produit des résultats relativement rapides et positifs.

\section{Le statut du komi à la lumière des enquêtes sociologiques}

En 2003, le ministère des questions nationales a lancé un suivi ethnolinguistique et sociologique pour mettre en évidence les problèmes concernant l'utilisation du komi sur le territoire de la République. Des enquêtes sociologiques sur un échantillon de population ont été réalisées en 2003 et $2006^{5}$. Les chercheurs voulaient savoir comment le komi était utilisé dans la vie quotidienne, comment il était perçu par la population non komie et comment son statut social était évalué par les différents groupes de population. Ils ont pu également mettre en évidence le degré d'attachement de la population à l'égard de la langue komie. Dans une situation où la quasi-totalité de la population de la République maîtrise le russe (d'après le recensement de 2002, 98 \% des Komis [Nacional'nyj 2005, p. 32]), les moyens permettant à la langue komie de conserver un statut élevé sont très limités.

Les enquêtes ont mis en évidence parmi les Komis une baisse du niveau de compétence active en komi (maîtrise de l'oral). La structure de la compétence se modifie: la proportion de personnes possédant une connaissance parfaite de la langue (c'est-à-dire qui sont capables de la parler, de la lire et de l'écrire) se réduit, tandis que la proportion de celles dont la compétence se limite à la compréhension augmente. Cette situation s'explique par le processus continu d'assimilation, mais aussi par la réduction naturelle de la génération qui avait été scolarisée en komi. 

du komi dans la vie publique, le degré d'attachement de l'ensemble de la population à l'égard de cette langue est assez élevé. Ainsi en 2006, parmi les Russes, dont seule une faible proportion ( 5 ou $6 \%$ ) parle couramment le komi, $34 \%$ ont déclaré souhaiter que leurs enfants et petits-enfants possèdent une connaissance active de cette langue (voir tableau 2).

\begin{tabular}{|l|c|c|c|}
\hline \multicolumn{2}{|l|}{$\begin{array}{l}\text { Souhaitez-vous ou non que vos enfants ou vos petits-enfants connaissent le } \\
\text { komi? }\end{array}$} & \multicolumn{3}{|c|}{ Total } & Russes & Komis \\
\hline & 21,7 & 14,4 & 43,6 \\
\hline 1. Oui, je souhaite qu'ils sachent parler et écrire le komi. & 23,8 & 19,9 & 36,2 \\
\hline 2. Oui, je souhaite qu'ils sachent parler et comprendre le komi. & 17,0 & 18,5 & 12,3 \\
\hline 3. Oui, mais il leur suffit de comprendre le komi. & 26,9 & 34,3 & 4,3 \\
\hline 4. Non, je ne vois pas la nécessité pour eux de connaître le komi. & 10,6 & 12,8 & 3,7 \\
\hline 5. Je ne sais pas répondre. & 100,0 & 100,0 & 100,0 \\
\hline Total & & &
\end{tabular}

Tableau 2. Opinion des Russes et des Komis sur leur désir que leurs enfants ou petits-enfants connaissent le komi (d'après les résultats de l'enquête de 2006, en pourcentage des personnes interrogées).

Le komi est présent à égalité avec le russe dans la signalétique des rues et des routes, ainsi que sur les plaques des établissements publics (conformément à la loi sur les Langues officielles). Cette pratique est approuvée par $65 \%$ des Komis et $49 \%$ des Russes. La présence d'informations internes en komi dans les lieux publics (magasins, gares, établissements publics) est soutenue par $54 \%$ des Komis et $40 \%$ des Russes. Compte tenu de la faible utilisation réelle du komi dans la sphère publique, les résultats de l'enquête révèlent un degré élevé d'attachement à l'égard de la langue komie, ce qui permet d'affirmer que le komi joue un rôle symbolique important dans l'identité régionale.

L'un des principaux moyens permettant d'assurer la conservation de la langue est l'enseignement de celle-ci dans les écoles. Le komi est, à l'heure actuelle, enseigné dans les écoles de la République des Komis en tant que langue maternelle (principalement dans les écoles rurales) et en tant que langue (officielle) non maternelle (de façon analogue aux langues étrangères). Mais le désir d'étudier le komi à l'école en tant que matière obligatoire est faible, y compris parmi les Komis. Cela s'explique par le fait que le komi est non seulement cantonné dans le domaine de la vie quotidienne, mais aussi supplanté dans les programmes scolaires par des matières plus populaires et par les langues étrangères. Alors qu'en $200634 \%$ des Russes et $80 \%$ des Komis souhaitaient que leurs enfants et petits-enfants possèdent une connaissance active du komi, seuls 
$27 \%$ des Russes et $58 \%$ des Komis étaient favorables à l'enseignement obligatoire du komi dans les écoles en tant que langue nationale. Et cette fracture ne cesse de s'élargir.

31 La question du statut du komi inquiète au plus haut point l'élite intellectuelle autochtone. À cet égard, il est intéressant d'étudier la façon dont les étudiants komis en lettres et sciences humaines, qui constituent le noyau de l'élite culturelle, perçoivent le statut de leur langue. Une enquête a été réalisée en vue de mettre en évidence leur évaluation du prestige et de l'importance sociale du komi'

Dans un premier temps, les problèmes ont été répertoriés conformément à la conception sociologique selon laquelle, dans le monde contemporain, les individus détiennent, d'après Bourdieu, diverses formes de capital : capital économique, social, culturel, symbolique, etc. Les aptitudes (ressources) constituent un capital fondamental, qui rapporte un certain « revenu ", de même que l'argent investi dans un secteur de production rapporte à son propriétaire un revenu matériel. Plus une ressource rapporte un revenu élevé, plus la demande sociale de cette ressource est forte. Ainsi, une bonne connaissance de la langue officielle est incontestablement une ressource essentielle pour atteindre un niveau économique et un statut élevés. Si la langue d'un petit peuple acquiert dans un territoire autonome un statut officiel, son importance en tant que ressource doit également augmenter.

Les personnes interrogées lors de l'enquête étaient étudiants à la faculté des lettres de l'Université d'État de Syktyvkar (spécialité "philologie nationale»), à l'Institut pédagogique national komi (spécialité "professeur des écoles", avec spécialisation complémentaire "professeur de langue et littérature komies dans l'enseignement secondaire fondamental») et au Collège pédagogique littéraire I. A. Kuratov de Syktyvkar (spécialité "enseignement dans les classes élémentaires", avec spécialisation en «enseignement de la langue et de la lecture komies à l'école élémentaire »). L'enquête a été réalisée auprès de 223 personnes.

L'écrasante majorité des personnes interrogées (94,6 \%) étaient des jeunes filles, la part des garçons n'était que de 5,4\%. Du point de vue de l'appartenance nationale, $95 \%$ se considéraient comme Komis et $5 \%$ comme Russes. $71,7 \%$ avaient pour langue maternelle le komi, et 24,7 \% le komi et le russe à égalité.

La question principale de l'enquête était: "Connaître le komi confère-t-il du prestige?» Les sondés avaient la possibilité de répondre de façon univoque à la question (par «oui» ou par «non») et/ou d'expliquer leur point de vue sous forme libre sur une feuille séparée.

36 La majorité des étudiants ( $52 \%$ ) ont répondu que la connaissance du komi était source de prestige. Mais comment comprenaient-ils la notion de prestige de la langue ? La réponse à cette question a pu être trouvée en analysant le contenu des opinions écrites.

Le plus souvent (dans $28,9 \%$ des cas), le prestige de la connaissance du komi est rattaché à l'existence de la République des Komis en tant que sujet de la Fédération de Russie, qui confère à la langue un poids particulier. Dans une telle situation, la langue acquiert un statut politique, devient en quelque sorte un symbole de l'appartenance à la communauté régionale. C'est pourquoi on lit souvent, parmi les réponses, des affirmations telles que: "C'est une honte de ne pas connaître la langue du peuple komi », «Puisque nous habitons dans la République des Komis, connaître le komi est 
source de prestige» ou «Tous les habitants de la République des Komis devraient connaître le komi. »

En seconde position (20\%), on trouve le point de vue selon lequel « connaître sa langue maternelle apporte du prestige ». Indéniablement, la connaissance de sa langue maternelle renforce l'identification et donne un sens particulier aux choix professionnels des personnes interrogées. Les étudiants expliquent : " Je suis fier de ma langue maternelle, je la parle dans la vie quotidienne, cela ne me gêne pas de parler komi dans les lieux publics ", "Il ne faut pas avoir honte de sa langue maternelle», "Nous devons être fiers d'avoir notre propre langue", "Connaître notre langue maternelle, c'est notre fierté ».

En troisième position (16,7\%), le prestige de la connaissance du komi est lié à la foi un peu irrationnelle dans l'idée que la connaissance de toute autre langue en plus du russe accroît le capital culturel d'une personne, témoigne d'une certaine richesse spirituelle et intellectuelle: "Il est prestigieux d'être bilingue ", "Plus on connaît de langues, mieux c'est", "À notre époque, les gens qui connaissent plusieurs langues sont davantage recherchés par les employeurs", "La connaissance de toute langue finit toujours par être utile». Dans cette croyance irrationnelle qu'il est prestigieux d'être bilingue réside une ressource potentielle de la langue. Par exemple, de nombreux étudiants pensent qu'en dehors de la République des Komis (dans les autres régions de Russie), le komi est considéré comme une langue étrangère, ce qui accroît la ressource intellectuelle des locuteurs de cette langue. Certains pensent que cela augmente leurs chances de trouver du travail. L'appartenance du komi à la famille des langues finnoougriennes fait naître le sentiment d'un lien avec le monde extérieur.

La quatrième raison $(15,6 \%)$ du prestige de la langue komie est son importance en tant que ressource professionnelle pour un individu. Ainsi, certains écrivent: «La connaissance du komi m'a aidé à choisir un métier, a élargi mes possibilités professionnelles ». Ils se déclarent convaincus que " cela peut aider aussi plus tard à avoir des perspectives professionnelles intéressantes ». En même temps, les étudiants ont conscience du caractère limité de leurs débouchés potentiels. En effet, il n'existe pas beaucoup de postes dans la République nécessitant la connaissance du komi.

41 Les sondés distinguent dans le prestige de la langue komie un aspect individuel, en particulier pour eux-mêmes en tant qu'étudiants, et un aspect social. L'importance sociale du komi, comme on pouvait le supposer, a été jugée faible. $47,5 \%$ des personnes interrogées ont estimé qu'elle était « insuffisante », et 17 \% qu'elle était «faible ». Peutêtre pour cette raison, près d'un tiers doutent que leurs enfants auront une connaissance du komi aussi bonne que la leur. À la question portant sur les perspectives de la langue komie au sein de la République, 33,2\% ont répondu que la langue "disparaîtrait progressivement", et $41,7 \%$ qu'elle "resterait au niveau actuel ».

42 La faible importance sociale du komi est confirmée par l'évaluation insuffisante des perspectives professionnelles qu'elle ouvre: l'enseignement du komi dans les écoles (comme langue maternelle ou non), la recherche scientifique et la littérature en komi n'ont qu'une ampleur très limitée; de même, rares sont ceux qui peuvent trouver un emploi avec le komi à la télévision, à la radio (il n'y a que très peu de postes), et il est tout à fait exceptionnel que la connaissance du komi soit exigée pour occuper un poste de fonctionnaire. 
Si le prestige du komi pour les étudiants eux-mêmes ne fait aucun doute, sur les plans professionnel, culturel, spirituel et identitaire, son prestige social est érodé, limité dans l'espace, et écartelé entre deux réalités : celle dans laquelle le statut de la langue est élevé, et une autre dans laquelle ce statut est bas. L'une des personnes interrogées écrit : «Cela dépend dans quelle compagnie l'on se trouve : si l'on est en compagnie de gens qui connaissent et travaillent avec la langue et la littérature komies, alors elle [la langue komie] est prestigieuse, mais si les gens ne sont pas du tout liés à la langue komie, alors elle ne l'est pas du tout. Dans les lieux publics, les gens qui entendent une conversation en komi se retournent et vous jettent des regards réprobateurs. "

Les étudiants sont nombreux à penser qu'il est mal vu de parler komi dans les lieux publics. L'enquête a confirmé l'hypothèse selon laquelle le sentiment de honte de nombreux étudiants parlant komi est dû au fait que, dans la conscience sociale, le komi est associé à la ruralité (la majorité de la population komie de la République habite à la campagne). Comme le niveau de vie à la campagne est incomparablement plus bas qu'en ville, le statut des ruraux est socialement moins valorisé.

L'existence de ces deux réalités est perçue assez nettement par les étudiants, mais fait l'objet d'évaluations légèrement différentes. Les personnes interrogées élaborent des stratégies d'interaction différentes dans leurs contacts avec chacune de ces deux réalités. En témoigne par exemple l'affirmation suivante: "Connaître le komi est prestigieux. Quand je regarde la télé avec des amis russes, c'est agréable. On regarde la chaîne komie et mes amis me demandent de traduire, et je le fais volontiers. » Autre affirmation: «De nos jours, ce n'est pas prestigieux, parce que les gens qui parlent en komi sont une offense pour les autres. C'est très gênant, et beaucoup de gens n'osent pas parler leur langue maternelle. » Le choix ou le rejet d'une stratégie de coopération dépend apparemment de la vision du monde des étudiants, de leur éducation, et de leur expérience personnelle de l'interaction.

La question du statut officiel et social du komi doit être envisagée dans le contexte de l'histoire de l'évolution du peuple komi au $\mathrm{xx}^{\mathrm{e}}$ siècle, processus dont elle est indissociable. Dans les années 1920, le komi remplissait réellement toutes les fonctions sociales sur le territoire autonome des Komis : plus de $90 \%$ de la population parlait le komi, et la politique du gouvernement central soutenait l'utilisation de la langue locale dans la pratique sociale et politique. Avec les changements politiques dans le pays, mais aussi du fait des changements démographiques au sein de la République, le statut social du komi est devenu peu à peu purement formel. Le russe occupe depuis longtemps la place principale dans tous les aspects de la vie de la République, et la population komie est devenue bilingue.

7 À la suite des mutations du début des années 1990, le komi a été rétabli au rang de langue officielle. Mais cela s'est produit alors que les conditions démographiques, linguistiques et culturelles étaient déjà en train de changer. L'enquête a mis en évidence que le komi au sein de la République ne fait pas l'objet d'une demande, comme le voudrait son statut de langue officielle. La question reste ouverte de savoir dans quelle mesure il est possible de modifier le système de soutien au statut officiel du komi dans une situation où son importance sociale se réduit.

Les enquêtes ont montré qu'un facteur essentiel pour préserver le statut de langue officielle, et par là même les mesures de soutien à la langue au niveau de la République, est le statut même de la République en tant que sujet de la Fédération de Russie. Des possibilités résident également dans le soutien et le développement de l'enseignement 
du komi à l'école en tant que langue maternelle et non maternelle, et dans l'élargissement de l'emploi du komi dans la sphère professionnelle. Le komi demeure également un symbole culturel de l'identité régionale. L'élévation de l'importance du komi en tant que ressource est indéniablement du ressort des autorités politiques de la République.

\section{BIBLIOGRAPHIE}

CYPANOV 2006 = ЦЫПАНОВ Е.А., «О РЕАЛИЗАЦИИ ЗАКОНА О ЯЗЫКАХ В РЕСПУБЛИКЕ КОМИ», ИСТОРИЯ И СОВРЕМЕННОЕ РАЗВИТИЕ РЕСПУБЛИКИ КОМИ В СОСТАВЕ РОССИЙСКОЙ ФЕДЕРАЦИИ: СБ. МАТЕРИАЛОВ НАУЧН. КОНФ., СЫКТЫВКАР, С. 150-166.

NACIONAL'NYJ 2005 = НАЦИОНАЛЬНЫЙ СОСТАВ НАСЕЛЕНИЯ И МЕЖНАЦИОНАЛЬНЫЕ ОТНОШЕНИЯ В РЕСПУБЛИКЕ КОМИ, АНАЛИТИЧЕСКАЯ ЗАПИСКА, ИЮЛЬ, ТЕРРИТОРИАЛЬНЫЙ ОРГАН ФЕДЕРАЛЬНОЙ СЛУЖБЫ ГОСУДАРСТВЕННОЙ СТАТИСТИКИ ПО РЕСПУБЛИКЕ КОМИ, СЫКТЫВКАР.

OSIPOV 1929 = ОСИПОВ С., «КОРЕНИЗАЦИЯ СОВЕТСКОГО АППАРАТА», ЮГЫД ТУЙ, ИЮНЬ.

POPOV \& NAPALKOV 2004 = ПОПОВ А.А., НАПАЛКОВ А.Д., «КОММЕНТАРИЙ К ПРИНЯТИЮ КОНСТИТУЦИИ КОМИ АССР 1937 ГОДА», ТВОИ КОНСТИТУЦИИ, РЕСПУБЛИКА, СБОРНИК ТЕКСТОВ КОНСТИТУЦИЙ КОМИ АССР И РЕСПУБЛИКИ КОМИ (1937-2003), СЫКТЫВКАР, С. 33-39.

POPOV \& NESTEROVA 2006 = ПОПОВ А.А., НЕСТЕРОВА Н.А., НАЦИОНАЛЬНЫЙ ВОПРОС В РЕСПУБЛИКЕ КОМИ В КОНЦЕ ХХ ВЕКА, СЫКТЫВКАР.

POPOV 2004 = ПОПОВ А.А., «ЗЫРЯНИЗАЦИЯ», ИСТОРИЯ КОМИ С ДРЕВНЕЙШИХ ВРЕМЕН ДО КОНЦА ХХ ВЕКА, Т. 2., СЫКТЫВКАР, С. 296-300.

TVOI $2004=$ ТВОИ КОНСТИТУЦИИ, РЕСПУБЛИКА. СБОРНИК ТЕКСТОВ КОНСТИТУЦИЙ КОМИ АССР И РЕСПУБЛИКИ КОМИ (1937-2003), СЫКТЫВКАР, 2004.

\section{NOTES}

1. En russe: КОМИ ОБЛАСТНОЙ КОМИТЕТ РОССИЙСКОЙ КОММУНИСТИЧЕСКОЙ ПАРТИИ (Большевиков) « Comité de l'oblast komi du Parti communiste de Russie (bolchevik)».

2. En russe : ОБЛАСтНОЙ ИСПОЛНИТЕЛЬНЫЙ кОмИТЕТ - l'instance la plus élevée du pouvoir exécutif sur le territoire du district autonome komi.

3. Ce terme désigne l'introduction de la langue komie (zyriène) dans la vie publique et politique du district, version komie de la politique d'indigénisation (коренизАция).

4. La région autonome komie fut transformée en République socialiste soviétique autonome komie (RSSA komie) en 1936.

5. Le groupe de recherche était composé en 2003 de R. I. Zubova, A. K. Konjuhov, O. Ju. Kuzivanova, N. S. Sergieva, E. A. Cypanov et en 2006 de A. K. Konjuhov et O. Ju. Kuzivanova. 1295 personnes ont été interrogées. Les résultats de l'enquête ont été publiés partiellement dans 
l'ouvrage Finno-Ugric Ethnicities in Russia: Yesterday, Today, and Tomorrow, Alexey K. Konuykhov, ed., Syktyvkar, 2009, pp. 154-188.

6. Enquête réalisée en mars-avril 2009 par O. Ju. Kuzivanova et M. S. Fedina.

\section{RÉSUMÉS}

Cet article dresse un aperçu historique de la question du statut officiel de la langue komie au sein de la République des Komis (Fédération de Russie) et évalue la situation ethnolinguistique actuelle. La période couverte va des années 1920, qui ont vu le komi occuper une position dominante sur le territoire autonome des Komis, jusqu'à la fin du xxe siècle, où le komi, en raison de toute une série de circonstances, a perdu sa position sociale. Ce travail s'appuie sur des sources historiques, des données démographiques et les résultats d'enquêtes sociologiques. Il s'interroge sur les ressources sociales qui permettraient de préserver la position du komi dans une situation où les Komis sont minoritaires, bilingues russe-komi et où le statut social du komi est bas.

The article reveals the historical aspects of the question of the Komi language's official status in the Komi Republic (Russian Federation) and analyses the present ethno-linguistic situation. The article covers the period from 1920-s, when the Komi language had a dominant position in the Komi autonomy, to the end of 20th century when the public positions were lost for a number of reasons. The authors use historical documents, demographical data and the results of sociological researches. The article puts the question about the possible resources for the preservation of Komi language's positions in the conditions of Komi people's minority, bilingualism and low public status of Komi language.

В СТАТЬЕ РАСКРЫВАЕТСЯ ИСТОРИЯ ВОПРОСА ГОСУДАРСТВЕННОГО СТАТУСА КОМИ ЯЗЫКА В РЕСПУБЛИКЕ КОМИ (РОССИЙСКАЯ ФЕДЕРАЦИЯ) И ДАЁТСЯ ОЦЕНКА СОВРЕМЕННОЙ ЭТНОЛИНГВИСТИЧЕСКОЙ СИТУАЦИИ. ХРОНОЛОГИЧЕСКИ ОХВАТЫ-ВАЕТСЯ ПЕРИОД С 1920-Х ГГ., КОГДА КОМИ ЯЗЫК НА ТЕРРИТОРИИ КОМИ АВТО-НОМИИ ЗАНИМАЛ ЛИДИРУЮЩИЕ ПОЗИЦИИ, ДО КОНЦА ХХ В., КОГДА КОМИ ЯЗЫК В СИЛУ РЯДА ОБСТОЯТЕЛЬСТВ ПОТЕРЯЛ ОБЩЕСТВЕННЫЕ ПОЗИЦИИ. АВТОРЫ ИС-ПОЛЬ ЗУЮТ ИСТОРИЧЕСКИЕ ДОКУМЕНТЫ, ДЕМОГРАФИЧЕСКИЕ ДАННЫЕ, РЕЗУЛЬ-ТАТЫ СОЦИОЛОГИЧЕСКИХ ИССЛЕДОВАНИЙ. СТАВИТСЯ ВОПРОС О ВОЗМОЖНЫХ СОЦИАЛЬᄀНЫХ РЕСУРСАХ СОХРАНЕНИЯ ПОЗИЦИЙ КОМИ ЯЗЫКА В УСЛОВИЯХ МАЛОЧИС ᄀЛЕНᄀНОСТИ КОМИ НАРОДА, КОМИ-РУССКОГО БИЛИНГВИЗМА КОМИ НАСЕЛЕНИЯ И НИЗКОГО ОБЩЕСТВЕННОГО СТАТУСА КОМИ ЯЗЫКА.

УДЖЫН ВИДЛАЛӦМА ИСТОРИЯ БОКСЯНЬ КОМИ РЕСПУБЛИКА МУТАСЫН (РОССИЯ ФЕДЕРАЦИЯ) КОМИ КЫВЛӦН КАНМУ ТӦДЧАНЛУН ДА ӦНіЯ КАДСЯ ЭТНОЛИНГВИСТИКА СИТУАЦИЯ. ХРОНОЛОГИЯ БОКСЯНЬ ШЫМЫРТӦМА КАДКОЛАСТ 1920 ВОЯССЯНЬ, КОР КОМИ КЫВ КОМИ АВТОНОМИЯ МУТАСЫН вӧлі МЕДшӧР КЫВЙӧН, ХХ НЭМ ПОМӧДЗ, КОР КОМИ КЫВ УНА ПОМКА ВӧСНА ВОШТіС АССЬЫС ТӦДЧАНЛУНСӧ. АВТОРЪЯ ВӧДИТЧӦНЫ ИСТОРИЯ ДОКУМЕНТЪЯС̈̈Н, ДЕМОГРАФИЯ

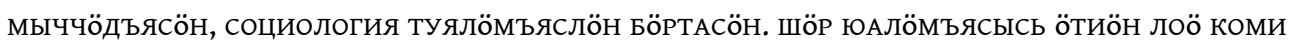

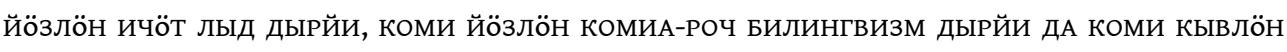
ВОЙТЫР КОСТЫН ИЧӦТ ТӦДЧАНЛУН ДЫРЙИ КОМИ КЫВЛЫСЬ ТӦДЧАНЛУНСӦ КЫПӦДЫСЬ ВЕРМАНА социАльнӧй позянлунъяс. 
INDEX

Mots-clés : statut de la langue, indigénisation, zyriénisation, bilinguisme, démographie nomsmotscles Komis, Russes

disciplines russe, komi

Index géographique: Komi (République), Fédération de Russie

Thèmes : histoire

Keywords : language status, indigenisation, zyrianisation, Bilingualism, Demography, Komi republic, Russia Federation, Komi, Russian, Russians 